Transpositions in short-term memory. Journal of Experimental Psychology, 1967, 74, 137-143.

NORMAN, D. A. Toward a theory of memory and attention. Psychological Review, 1968, 75, 522-536.

PETERSON, D. R., \& PETERSON, M. J. Short-term retention of individual verbal items. Journal of Experimental Psychology, 1959, 58 , 192-198.
NOTE

1. I wish to thank Mrs. Edith V. Sullivan for he assistance in collecting and analyzing the data and Dr. Michael T. Turvey for his comments upon the the manuscript and for stimulating my in terest in short-term memory. This research was supported by a grant from the University of Connecticut Research Foundation.

\title{
Effect of simultaneous presentation of conceptually similar and dissimilar items on paired associate learning ${ }^{1}$
}

\begin{abstract}
PAUL S. SHERMAN and SAM C. BROWN, Kansas State University, Manhattan, Kans. 66502
\end{abstract}

Paired-associate learning was retarded significantly when subsets of conceptually dissimilar pairs were presented simultaneously rather than successively for learning. The retardation was eliminated, however, when the subsets contained pairs sharing similar stimulus terms or similar response terms. Under both methods of presentation, learning was faster when pairs were grouped on the basis of conceptual similarity than when the same pairs were presented in dissimilar groupings.

The purpose of the present research was to determine the effect on paired-associate (PA) learning of (a) blocking similar and dissimilar subsets of pairs within the list for sequential presentation, and (b) presenting pairs within each block either successively (one at a time) or simultaneously (all together) for learning. Previous research (Brown \& Brown, 1965; Brown \& Read, 1966) showed that simultaneous presentation of more than one pair enabled Ss to categorize the pairs into subsets, but that such categorization retarded PA learning significantly when compared with the standard, successive method of pair presentation. In the earlier research, conceptually unrelated items were blocked together for simultaneous presentation. Research by Rotberg \& Woolman (1963) has shown that blocked, successive presentation of related materials produced better PA performance than unblocked presentation. On the basis of this finding, it was predicted that the deleterious effect of simultaneous presentation found earlier should be reduced or eliminated if the blocking of pairs were based on some organizational principle. Consequently, the present study compared simultaneous and successive methods of pair presentation under conditions where pairs grouped for contiguous presentation were conceptually similar or dissimilar. To obtain more general information on the effects of locus of similarity, pairs were grouped on the basis of stimulus or response term similarity.

\section{METHOD}

The 128 college student Ss practiced on an identical list of 16 paired associates for 20 trials. The list was exactly the list used previously by Underwood \& Schultz (1961; List 5). The 16 stimulus (S) terms consisted of four categories of four words each. Each category represented a different conceptual class of words (male first names, animals, dances, and clergymen). The 16 response (R) members represented instances of four different categories of four words each (diseases, sports, countries, and chemical elements). The pairs were constructed by pairing the S-terms in each conceptual category with R-terms from different categories (e.g., Bob-Nitrogen; Bill-Sparrow; Joe-Germany; John-Cancer).
Learning for all Ss was carried out under the recall (pairing-test) procedure. Four of the eight groups of $16 \mathrm{Ss}$ each learned under a successive method (individual pair presentation on pairing and test series) and four under a simultaneous method (blocks of four pairs grouped together for simultaneous exposure on pairing and test series). The four simultaneous groups differed with respect to the kind of pair groupings used (conceptually organized or unorganized). For Group SO (S-term organization), the four pairs within each block contained $S$ terms from the same conceptual category and $R$ terms from different categories. With Group RO (R-term organization), each block of four pairs consisted of $R$ terms from the same category and $S$ terms from different categories. Within each list ( $S O$ and $R O$ ), the same pairs were grouped together on each trial. The two remaining simultaneous groups learned unorganized blocks of pairs, i.e., each block of four pairs presented for simultaneous exposure contained $S$ and $R$ terms from different conceptual categories. For Group UC (unorganized-constant grouping), the same four dissimilar pairs were grouped together on every trial, following the procedure for Groups SO and RO. In Group UV (unorganized-varied grouping), the pairs within each block changed from trial to trial. Thus, conceptual organization was varied by using four lists of the same $S-R$ pairs which differed only in the temporal order of presentation of these pairs in the list.

Under the successive method of pair presentation, each of four groups learned exactly one of the four list orders used by each of the simultaneous groups. Consequently, the pairs were blocked in the same way under both methods of pair presentation. However, with the successive method, the pairs within each block were exposed individually.

The list members were presented on slides either individually (16 slides) or vertically in blocks of four (4 slides) under the successive and simultaneous methods, respectively. Corresponding slides were produced for S-terms. A group-data collection procedure was used wherein all $16 \mathrm{Ss}$ in each group served in a single session. Each trial began with the successive presentation of the 4 or 16 slides containing the pairs, through a Kodak slide projector, followed by the successive exposure of corresponding slides containing the S-terms, during which Ss were asked to write down in answer booklets the correct R-term for each S-term. Rate of presentation of the slides on the pairing part of each trial was 2 and $8 \mathrm{sec}$, and on test series, 4 and $16 \mathrm{sec}$ for successive and simultaneous groups, respectively. The interval between pairing-test series and trials 
Table 1

Mean Number of Errors for Each Group

\begin{tabular}{lccc} 
& \multicolumn{3}{c}{ Method of Presentation } \\
\cline { 3 - 4 } List & Simultaneous & Successive & Total \\
\hline Organized & & & \\
SO & 45.2 & 50.3 & 47.8 \\
RO & 56.1 & 44.8 & 50.4 \\
Total & 50.6 & 47.6 & 49.1 \\
Unorganized & & & \\
UC & 86.8 & 43.9 & 65.3 \\
UV & 87.6 & 63.2 & 75.4 \\
Total & 87.2 & 53.5 & 70.4 \\
\hline
\end{tabular}

was $8 \mathrm{sec}$. Order of the items within a block, as well as the order in which the blocks appeared, was changed unsystematically from trial to trial, although the same order of presentation was used on pairing and test series within trials.

\section{RESULTS}

Table 1 shows the mean number of total errors in learning for each experimental group. As can be seen, the effect of method of pair presentation varied depending upon whether the lists were organized or unorganized. For unorganized lists (Groups UC and UV), more errors were made under the simultaneous than under the successive method; for organized lists (Groups SO and RO), overall performance was practically identical for the two methods, leading both to a significant main effect of method and Method by Organization interaction (Fs $>4.00$, df $=1 / 124, \quad p<.05$; the criterion of significance for all analyses). Significantly fewer overall errors also occurred for groups learning organized than unorganized lists $(F=7.97, \mathrm{df}=1 / 124)$. Only performance by the successive UC group equaled that of the organized groups. Separate analyses based upon SO and RO groups and upon $U C$ and $U V$ groups revealed only the main effect of method significant for unorganized groups $(F=7.82$, $\mathrm{df}=1 / 60$ ). All other main effects and interactions in both analyses were nonsignificant.

When items are grouped into subsets for presentation, overt substitution errors can be responses from within the same subset (within-errors) or from other subsets (between-errors). In the previous research, it was shown that simultaneous presentation produced significantly more within- than between-errors, relative to the opportunities for each type of error to occur. The same analysis performed on the present data revealed a significantly greater percentage of within-errors under the simultaneous(37.2) than under the successive (29.5) method, and, conversely, more between-errors under the successive (70.5) than under the simultaneous (62.8) method $(F=4.50$, $\mathrm{df}=1 / 115)$. Furthermore, the magnitude of the differences was nearly equivalent under each of the four lists, as indicated by a nonsignificant Method by Lists in teraction. It should be noted, however, that more total numbers of both within- and betweenerrors were made under the simultaneous (4.0 and 19.5 , respectively) than successive ( 2.2 and 14.6, respectively) method when the list was unorganized. This was not true with organized lists. The simultaneous groups made more within-errors (9.4) than did successive groups $(8.0)$, while successive groups produced more between-errors (8.3) than did simultaneous groups(6.1).

To help identify the source of interference in learning unorganized as compared with organized lists, a tabulation was made of the percentage of similar- and dissimilar-errors committed during learning. More specifically, two types of similar-errors were identified. One was a substitution of an incorrect response conceptually similar to the correct response (R-errors), and the other was a response paired with an $S$ term similar to the correct $\mathrm{S}$ term (S-errors). All substitutions which were not conceptually similar either to the correct $R$ or $S$ term were designated as dissimilar-errors. For S-, R-, and dissimilar-errors, respectively, the mean percentages were: for List SO-49.8, 29.7, and 20.5; for List RO-29.5, 56.8, and 13.7; and for unorganized lists-44.7, 23.7, and 31.6. As indicated, there were relatively more similar-errors (S- and R-errors combined) in List SO (79.5) than in unorganized lists (68.4), and conversely, more dissimilar-errors in unorganized lists than in List SO. Both differences were significant $(F=7.53, \mathrm{df}=1 / 90)$. Likewise, the percentage of dissimilar-errors in unorganized lists was significantly greater than the percentage in List $\mathrm{RO}(\mathrm{F}=20.26$, $\mathrm{df}=1 / 92)$. However, the reduction in dissimilar-errors in List RO was accompanied only by an increase in similar Rerrors; significantly more R-than Serrors occurred in List RO, while significantly more S- than Rerrors were committed in both SO and unorganized lists (ps<.05). All interactions involving type of unorganized list (UC and UV), as well as method of presentation, were nonsignificant.

\section{DISCUSSION}

Consistent with the findings of the previous research, the present results showed a significant decrement in PA performance when subsets of conceptually dissimilar pairs were grouped together for simultaneous presentation. However, as hypothesized, the decrement was eliminated when pairs within blocks were conceptually similar to one another. As suggested previously (Brown \& Brown, 1965; Brown \& Read, 1966), simultaneous presentation of groups of pairs may serve to strengthen interpair associations among items within the subset, thereby increasing response confusion during testing. The occurrence of more within-subset errors under the simultaneous than under the successive method, both with organized and unorganized lists, is consistent with such an interpretation, as well as with previous findings (Brown \& Brown, 1965; Brown \& Read, 1966; Rotberg \& Woolman, 1963). However, with organized lists, there was no decrement from simultaneous presentation. This suggests that the negative effects of within-subset interference were counteracted by a decrease in between-subset interference, as indicated by the fewer absolute numbers of between-errors under the simultaneous than under the successive method. On the other hand, no such reduction in between-errors occurred with unorganized lists. Thus, simultaneous presentation of conceptually similar items enabled Ss to differentiate more easily between similar and dissimilar items, while simultaneous exposure of dissimilar items did not.

The occurrence of generally faster learning with blocks of pairs comprised of similar S-terms, as compared with blocks constituted entirely of dissimilar items, is consistent with the results of Rotberg \& Woolman (1963). However, the further observation of overall better PA performance with blocks of similar R-terms is not. One likely explanation for the discrepancy may involve a procedural difference between the two studies. In the Rotberg and Woolman study, the pairs were blocked for study but scrambled during testing (recall). In this study, pairs were blocked both during study and testing. Consequently, if $S$ could recall the correct response for beginning pairs in the subset, he could then delimit subsequent responses to items of the same conceptual category. That Ss probably used such a strategy is suggested by the large number of R-term substitution errors with the RO list.

\section{REFERENCES}

BROWN, S. C., \& BROWN, P. Comparison of successive and simultaneous methods of pair presentation in paired-associate learning. Psychonomic Science, 1965, 2, 309-310.

BROWN, S. C., \& READ, J. D. Effect of simultaneous list presentation on paired-associate learning. Psychological Reports, 1966, 19, 787-790.

ROTBERG, I. C., \& WOOLMAN, M. Verbal paired-associate learning as a function of grouping similar stimuli or responses. Journal of Experimental Psychology, 1963, 65, 47-51.

UNDERWOOD, B. J., \& SCHULZ, R. W. Studies of distributed practice: XXI. Effect of interference from language habits. Joumal of Experimental Psychology, 1961, 62, 571-575. NOTE

1. This investigation was supported by an NIMH Training Grant in Experimental Psychology (5 TO 1 MH-08359) and by Public Health Service Research Grant MH-11179. 